\title{
Certain Results on Ricci Solitons in $\alpha$-Sasakian Manifolds
}

\author{
S. R. Ashoka, C. S. Bagewadi, and Gurupadavva Ingalahalli \\ Department of Mathematics, Kuvempu University, Shankaraghatta, Shimoga, Karnataka 577 451, India
}

Correspondence should be addressed to C. S. Bagewadi; prof_bagewadi@yahoo.co.in

Received 1 April 2013; Revised 26 June 2013; Accepted 26 June 2013

Academic Editor: Giovanni Calvaruso

Copyright (C) 2013 S. R. Ashoka et al. This is an open access article distributed under the Creative Commons Attribution License, which permits unrestricted use, distribution, and reproduction in any medium, provided the original work is properly cited.

We study Ricci solitons in $\alpha$-Sasakian manifolds and show that it is a shrinking or expanding soliton and the manifold is Einstein with Killing vector field. Further, we prove that if $V$ is conformal Killilng vector field, then the Ricci soliton in 3-dimensional $\alpha$-Sasakian manifolds is shrinking or expanding but cannot be steady.

\section{Introduction}

A Ricci soliton $(g, V, \lambda)$ is a generalization of an Einstein metric and is defined on a Riemannian manifold $(M, g)$ by

$$
\mathscr{L}_{V} g+2 S+2 \lambda g=0
$$

where $V$ is a complete vector field on $M$ and $\lambda$ is a constant. The Ricci soliton is said to be shrinking, steady, or expanding according as $\lambda$ is negative, zero, and positive, respectively. Long-existing solutions, that is, solutions which exist on an infinite time interval, are the self-similar solutions, which in Ricci flow are called Ricci soliton.

Compact Ricci solitons are the fixed points of the Ricci flow $\partial g / \partial t=-2 \operatorname{Ric}(g)$ projected from the space of metrics onto its quotient modulo diffeomorphisms and scalings and often arise as blow-up limits for the Ricci flow on compact manifolds. If the vector field $V$ is the gradient of a potential function $-f$, then $g$ is called a gradient Ricci soliton and (1) assumes the form

$$
\nabla \nabla f=S+\lambda g
$$

A Ricci soliton on a compact manifold is a gradient Ricci soliton. A Ricci soliton on a compact manifold has constant curvature in dimension 2 [1] and also in dimension 3 [2]. In [3], Perelman proved that a Ricci soliton on a compact $n$ manifold is a gradient Ricci soliton. In [4], Sharma studied Ricci solitons in $K$-contact manifolds, where the structure field $\xi$ is Killing, and he proved that a complete $K$-contact gradient soliton is compact Einstein and Sasakian. In [5], Tripathi studied Ricci solitons in $N(k)$-contact metric and $(k, \mu)$ manifolds. In [6], Ghosh and Sharma studied $K$-contact metrics as Ricci solitons. In [7], Nagaraja and Premalatha studied Ricci solitons in $f$-Kenmotsu manifolds and 3dimensional trans-Sasakian manifolds. Recently, Bagewadi and Ingalahalli [8] studied Ricci solitons in Lorentzian $\alpha$ Sasakian manifolds. Motivated by the previous studies on Ricci solitons, in this paper, we study Ricci solitons in an $\alpha$ Sasakian manifolds, where $\alpha$ is some constant.

\section{Preliminaries}

Let $M$ be an almost contact metric manifold of dimension $n$ equipped with an almost contact metric structure $(\phi, \xi, \eta, g)$ consisting of a $(1,1)$ tensor field $\phi$, a vector field $\xi$, a 1-form $\eta$, and a Riemannian metric $g$, which satisfy

$$
\begin{gathered}
\phi^{2}=-I+\eta \otimes \xi, \quad \eta(\xi)=1, \quad \eta \circ \phi=0, \quad \phi \xi=0, \\
g(\phi X, \phi Y)=g(X, Y)-\eta(X) \eta(Y), \quad \eta(X)=g(X, \xi),
\end{gathered}
$$

for all $X, Y \in \mathfrak{X}(M)$. An almost contact metric manifold $M(\phi, \xi, \eta, g)$ is said to be $\alpha$-Sasakian manifold if the following conditions hold:

$$
\begin{gathered}
\left(\nabla_{X} \phi\right) Y=\alpha(g(X, Y) \xi-\eta(Y) X), \\
\nabla_{X} \xi=-\alpha \phi X, \quad\left(\nabla_{X} \eta\right) Y=\alpha g(X, \phi Y),
\end{gathered}
$$

for some nonzero constant $\alpha$ on $M$. 
In an $\alpha$-Sasakian manifold, we have the following relations:

$$
\begin{gathered}
R(X, Y) \xi=\alpha^{2}[\eta(Y) X-\eta(X) Y] \\
R(\xi, X) Y=\alpha^{2}[g(X, Y) \xi-\eta(Y) X] \\
\eta(R(X, Y) Z)=\alpha^{2}[g(Y, Z) \eta(X)-g(X, Z) \eta(Y)], \\
S(X, \xi)=\alpha^{2}(n-1) \eta(X) \\
S(\xi, \xi)=\alpha^{2}(n-1) \\
Q \xi=\alpha^{2}(n-1) \xi
\end{gathered}
$$

for all $X, Y, Z \in \mathfrak{X}(M)$, where $R$ is the Riemannian curvature tensor, $S$ is the Ricci tensor, and $Q$ is the Ricci operator.

\section{Ricci Solitons in $\alpha$-Sasakian Manifold}

In this section, we prove some theorems on Ricci solitons in $\alpha$-Sasakian manifold.

Proposition 1. A complete Einstein $\alpha$-Sasakian manifold is compact.

Proof. Let $M$ be a complete Einstein $\alpha$-Sasakian manifold, then the general form is given by

$$
S(X, Y)=\frac{r}{n} g(X, Y) \Longrightarrow Q=\frac{r}{n} I .
$$

Operating $\xi$ in (12) and using (11) show $r=n(n-1) \alpha^{2}$. Hence we get $Q=\alpha^{2}(n-1) I$. So the Ricci curvatures are equal to $\alpha^{2}(n-1)$ which is a positive constant. By Myers's theorem [9], we conclude that $M$ is compact.

Theorem 2. If the metric $g$ of an $\alpha$-Sasakian manifold $(M, g)$ is a gradient Ricci soliton, then the Ricci soliton is a shrinking soliton and $(M, g)$ is compact Einstein.

Proof. Equation (2) can be written as

$$
\nabla_{Y} D f=Q Y+\lambda Y
$$

where $D$ denotes the gradient operator of $g$ and $Y$ denotes an arbitrary vector field on $M$. Using this we derive

$$
R(X, Y) D f=\left(\nabla_{X} Q\right) Y-\left(\nabla_{Y} Q\right) X
$$

Taking its inner product with $\xi$, substituting $X=\xi$, and using (7) and (11), we have

$$
D f=(\xi f) \xi .
$$

Substituting (15) in (13), we get

$$
Y(\xi f) \eta(X)-\alpha(\xi f) g(\phi Y, X)=S(X, Y)+\lambda g(X, Y) .
$$

Interchanging $X$ and $Y$ in (16), we have

$$
X(\xi f) \eta(Y)-\alpha(\xi f) g(\phi X, Y)=S(Y, X)+\lambda g(Y, X) .
$$

Adding (16) and (17), we have

$$
X(\xi f) \eta(Y)+Y(\xi f) \eta(X)=2 S(Y, X)+2 \lambda g(Y, X) .
$$

Putting $Y=\xi$ in (18), we have

$$
X(\xi f)=\left[\alpha^{2}(n-1)+\lambda\right] \eta(X) .
$$

The use of the previous two equations provides

$$
S(X, Y)=\left[\alpha^{2}(n-1)+\lambda\right] \eta(X) \eta(Y)-\lambda g(Y, X) .
$$

Consequently, (13) assumes the form

$$
\nabla_{Y} D f=\left[\alpha^{2}(n-1)+\lambda\right] \eta(Y) \xi
$$

Using this, we compute $R(X, Y) D f$, and taking inner product with $\xi$ (bearing in mind that $D f=(\xi f) \xi)$, we obtain $\left[\alpha^{2}(n-\right.$ $1)+\lambda]=0$. Therefore, from (19), we have $X(\xi f)=0$; that is, $\xi f$ is constant or $\xi f=c$. Hence (15) can be written as $d f=c \eta$. Its exterior derivative implies $c d \eta=0$. Hence $c=0$. Thus $f$ is constant.

Consequently, (13) reduces to $S=\alpha^{2}(n-1) g$; that is, an $\alpha$-Sasakian manifold is an Einstein. Also, as $\lambda=-\alpha^{2}(n-1)$ is negative for $\alpha>0$ or $\alpha<0$; that is, Ricci soliton in $\alpha$-Sasakian manifolds is shrinking.

From above-mentioned theorem, we state the following corollary.

Corollary 3. If a metric $g$ of a compact $\alpha$-Sasakian manifold $(M, g)$ is a Ricci soliton, then $g$ is a shrinking soliton and the manifold is Einstein.

Theorem 4. If a metric $g$ in an $\alpha$-Sasakian manifold is a Ricci soliton with $V=\xi$, then it is Einstein.

Proof. Putting $V=\xi$ in (1), then we have

$$
\left(\mathscr{L}_{\xi} \mathfrak{g}+2 S+2 \lambda g\right)(X, Y)=0
$$

where

$$
\left(\mathscr{L}_{\xi} g\right)(X, Y)=g\left(\nabla_{X} \xi, Y\right)+g\left(X, \nabla_{Y} \xi\right)=0 .
$$

Substituting (23) in (22), then we get the result.

Proposition 5. If an $\alpha$-Sasakian manifold is a Ricci soliton with $V$ point-wise collinear with $\xi$, then $V$ is a constant multiple of $\xi$ and the manifold is Einstein.

Proof. From (1), we have

$$
\left(\mathscr{L}_{V} g\right)(X, Y)+2 S(X, Y)+2 \lambda g(X, Y)=0,
$$

where

$$
\left(\mathscr{L}_{V} g\right)(X, Y)=g\left(\nabla_{X} V, Y\right)+g\left(X, \nabla_{Y} V\right) .
$$

Substituting (25) in (24), then we obtain

$$
g\left(\nabla_{X} V, Y\right)+g\left(X, \nabla_{Y} V\right)+2 S(X, Y)+2 \lambda g(X, Y)=0 .
$$


Putting $V=a \xi$ in (26), we get

$$
(X a) \eta(Y)+(Y a) \eta(X)+2 S(X, Y)+2 \lambda g(X, Y)=0 \text {. }
$$

Putting $X=Y=\xi$ in (27), we have

$$
(\xi a)+\alpha^{2}(n-1)+\lambda=0 .
$$

Again putting $X=\xi$ in (27), we obtain

$$
(Y a)=\left[-\alpha^{2}(n-1)-\lambda\right] \eta(Y) .
$$

Equation (30) implies that

$$
d a=\left[-\alpha^{2}(n-1)-\lambda\right] \eta .
$$

Applying $d$ on both sides,

$$
d^{2} a=\left[-\alpha^{2}(n-1)-\lambda\right] d \eta
$$

Equation (31) implies that $d^{2} a=0$, but $d \eta$ is nowhere vanishing. Therefore, $-\lambda-\alpha^{2}(n-1)=0$ which implies $d a=0$; that is, $a$ is constant. As $\xi$ is Killing, we conclude that the manifold is Einstein which completes the proof.

Definition 6. A vector field $V$ is said to be conformal Killing vector field if it satisfies

$$
\mathscr{L}_{V} g=2 \rho g
$$

for some scalar function $\rho$.

Theorem 7. Let $(g, V, \lambda)$ be a Ricci soliton in an $\alpha$-Sasakian manifolds $(M, g)$. Then $(M, g)$ is Ricci-semisymmetric if and only if $V$ is conformal Killing.

Proof. Suppose that $V$ is a conformal Killing vector field, and from (1), we have

$$
2 \rho g(X, Y)+2 S(X, Y)+2 \lambda g(X, Y)=0 .
$$

The previous equation implies that

$$
S(X, Y)=[-\rho-\lambda] g(X, Y) .
$$

This shows that the Ricci soliton is Einstein as follows:

$$
Q X=[-\rho-\lambda] X
$$

Let $M$ be an $\alpha$-Sasakian manifolds; then we have [10]

(1) Einstein,

(2) locally Ricci symmetric,

(3) Ricci semisymmetric; that is, $R \cdot S=0$.

The implication $(1) \rightarrow(2) \rightarrow(3)$ is trivial. Now, we prove the implication $(3) \rightarrow(1)$.

Now,

$(R(X, Y) \cdot S)(U, V)=-S(R(X, Y) U, V)-S(U, R(X, Y) V)$.
Considering $R \cdot S=0$ and putting $X=\xi$ in (36), we have

$$
S(R(\xi, Y) U, V)+S(U, R(\xi, Y) V)=0 .
$$

By using (7) in (37), we obtain

$$
\begin{aligned}
& \alpha^{2}[g(Y, U) S(\xi, V)-\eta(U) S(Y, V)] \\
& \quad+\alpha^{2}[g(Y, V) S(U, \xi)-\eta(V) S(U, Y)]=0 .
\end{aligned}
$$

Putting $U=\xi$ in (38) and by using (3), (9), and (10) on simplification, we obtain

$$
S(Y, V)=(n-1) \alpha^{2} g(Y, V) .
$$

Substituting (39) in (1), we have

$$
\left(\mathscr{L}_{V} g\right)(X, Y)=\rho g(X, Y),
$$

where $\rho=2\left[-\alpha^{2}(n-1)-\lambda\right]$; that is, $V$ is conformal Killing.

Now we study Ricci solitons in 3-dimensional $\alpha$-Sasakian manifolds.

Theorem 8. In a 3-dimensional $\alpha$-Sasakian manifolds, a Ricci soliton $(g, V, \lambda)$, where $V$ is conformal Killing, is

(i) shrinking for $\rho=2 \alpha^{2}$,

(ii) expanding for $\rho<2 \alpha^{2}$ and $\rho>2 \alpha^{2}$.

Proof. Suppose that $(M, g)$ is a 3 -dimensional $\alpha$-Sasakian manifolds and $(g, V, \lambda)$ is a Ricci soliton in $(M, g)$. If $V$ is a conformal Killing vector field, then

$$
\mathscr{L}_{V} g=2 \rho g
$$

for some scalar function $\rho$.

In a 3 -dimensional $\alpha$-Sasakian manifolds and from (1), we have

$$
\begin{gathered}
S(X, Y)=[-\rho-\lambda] g(X, Y), \\
Q X=[-\rho-\lambda] X, \\
r=3[-\rho-\lambda] .
\end{gathered}
$$

In a 3-dimensional $\alpha$-Sasakian manifolds, the curvature tensor $R$ is given by

$$
\begin{aligned}
R(X, Y) Z= & g(Y, Z) Q X-g(X, Z) Q Y \\
& +S(Y, Z) X-S(X, Z) Y \\
& -\frac{r}{2}[g(Y, Z) X-g(X, Z) Y]
\end{aligned}
$$

Using (42), (43), and (44) in (45), we get

$$
R(X, Y) Z=\left\{2[-\rho-\lambda]-\frac{r}{2}\right\}[g(Y, Z) X-g(X, Z) Y]
$$


Putting $X=Z=\xi$ in (46), we get

$$
R(\xi, Y) \xi=\left\{2[-\rho-\lambda]-\frac{r}{2}\right\}[\eta(Y) \xi-Y] .
$$

In an $\alpha$-Sasakian manifolds $R(\xi, Y) \xi$ is given by

$$
R(\xi, Y) \xi=\alpha^{2}[\eta(Y) \xi-Y] .
$$

From (47) and (48), we have

$$
\left\{2[-\rho-\lambda]-\frac{r}{2}-\alpha^{2}\right\}[\eta(Y) \eta(W)-g(Y, W)]=0 .
$$

The previous equation implies that

$$
\left\{2[-\rho-\lambda]-\frac{r}{2}-\alpha^{2}\right\}=0 .
$$

From (44) and (50), we have

$$
\lambda=-\left[\rho+2 \alpha^{2}\right] .
$$

(i) If $\rho=2 \alpha^{2}$ implies $\lambda=-4 \alpha^{2}$, that is, $\lambda<0$. Hence Ricci soliton is shrinking.

(ii) Let $\rho<2 \alpha^{2}$, suppose $\rho=\left(-2 \alpha^{2}-1\right)<2 \alpha^{2}$ implies $\rho+2 \alpha^{2}=-1$, that is, $-\lambda=1>0$. Hence Ricci soliton is expanding.

(iii) Let $\rho>2 \alpha^{2}$ implies $-2 \alpha^{2}<-\rho$. If $-2 \alpha^{2}-2 \alpha^{2}<$ $-\rho-2 \alpha^{2}$ then $-4 \alpha^{2}<-\lambda$ implies $\lambda>4 \alpha^{2}$. Hence Ricci soliton is expanding.

If $\rho=0$ in (41), then $\mathscr{L}_{V} g=0$; that is, conformal vector field does not exist, and then the Ricci soliton is generalization of Einstein metric, then $V$ is a Killing vector field. On base of this condition, we state the following.

Remark 9. A Ricci soliton in $\alpha$-Sasakian manifolds is shrinking, if $V$ is a Killing vector field.

\section{Acknowledgment}

The authors are grateful to the referee for their valuable suggestions to improve this paper.

\section{References}

[1] R. S. Hamilton, "The Ricci flow on surfaces," in Mathematics and General Relativity, vol. 71 of Contemporary Mathematics, pp. 237-262, American Mathematical Society, 1988.

[2] T. Ivey, "Ricci solitons on compact three-manifolds," Differential Geometry and Its Applications, vol. 3, no. 4, pp. 301-307, 1993.

[3] G. Perelman, "The entropy formula for the Ricci flow and its geometric applications," http://arxiv.org/abs/math/0211159.

[4] R. Sharma, "Certain results on $K$-contact and $(k, \mu)$-contact manifolds," Journal of Geometry, vol. 89, no. 1-2, pp. 138-147, 2008.
[5] M. M. Tripathi, "Ricci solitons in contact metric manifolds," http://arxiv.org/abs/0801.4222.

[6] A. Ghosh and R. Sharma, "K-contact metrics as Ricci solitons," Beiträge zur Algebra und Geometrie, vol. 53, no. 1, pp. 25-30, 2012.

[7] H. G. Nagaraja and C. R. Premalatha, "Ricci solitons in fKenmotsu manifolds and 3-dimensional trans-Sasakian manifolds," Progress in Applied Mathematics, vol. 3, no. 2, pp. 1-6, 2012.

[8] C. S. Bagewadi and G. Ingalahalli, "Ricci solitons in Lorentzian $\alpha$-Sasakian manifolds," Acta Mathematica Academiae Paedagogicae Nyíregyháziensis, vol. 28, no. 1, pp. 59-68, 2012.

[9] S. B. Myers, "Riemannian manifolds with positive mean curvature," Duke Mathematical Journal, vol. 8, no. 2, pp. 401-404, 1941.

[10] G. Ingalahalli and C. S. Bagewadi, "Ricci solitons in $(\epsilon)$-TransSasakain manifolds," Journal of Tensor Society, vol. 6, no. 1, pp. 145-159, 2012. 


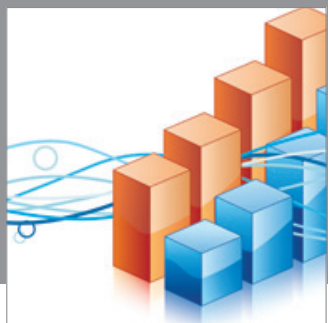

Advances in

Operations Research

mansans

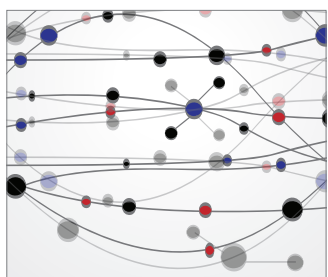

The Scientific World Journal
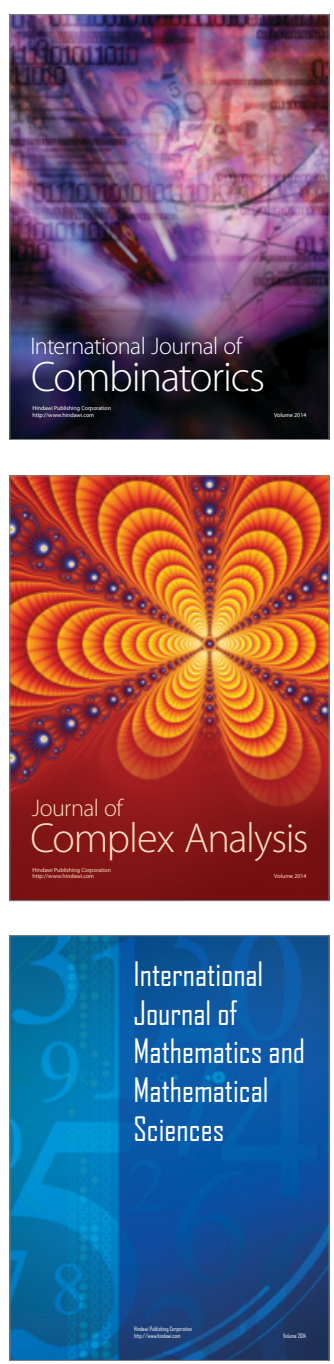
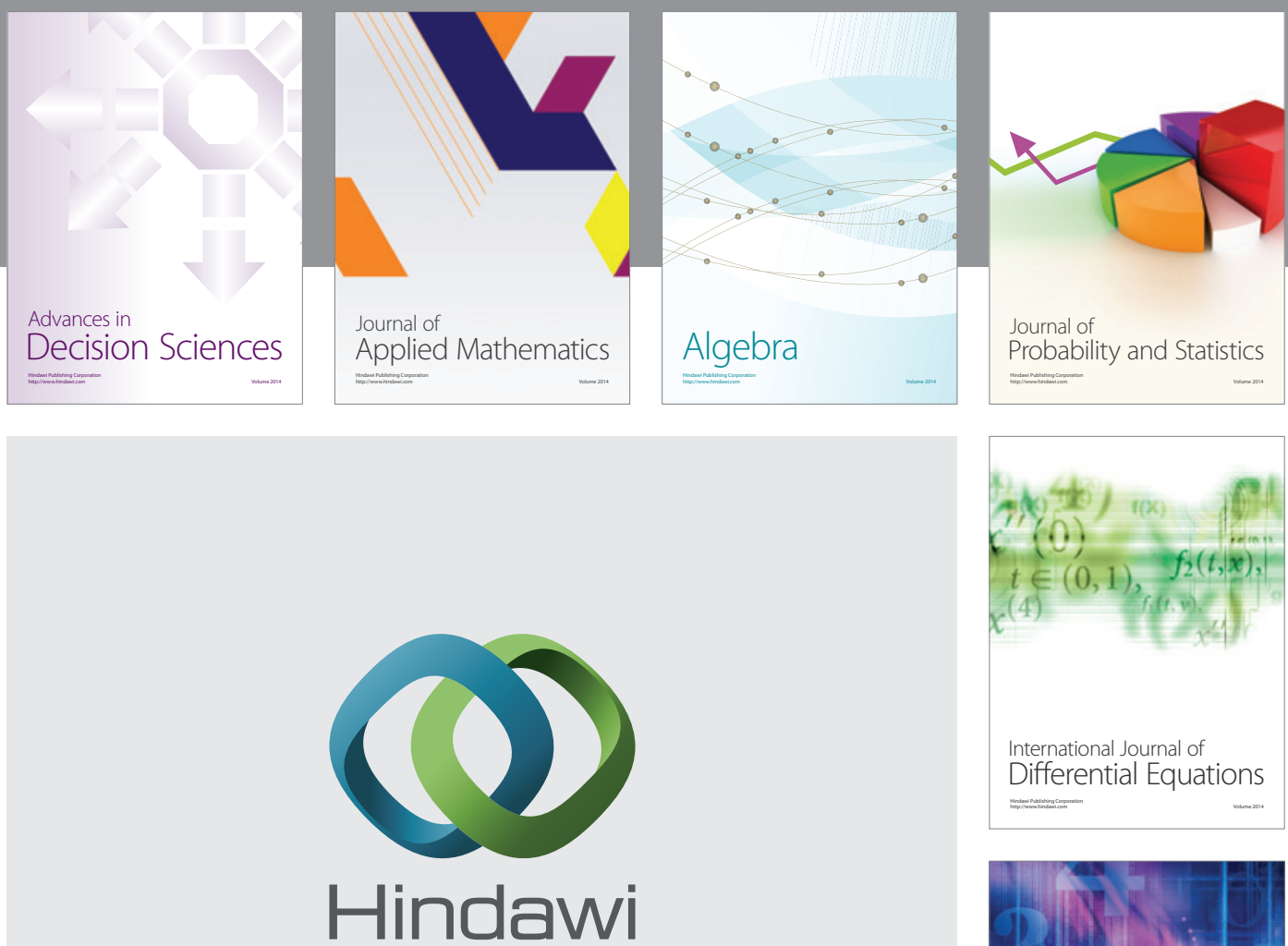

Submit your manuscripts at http://www.hindawi.com
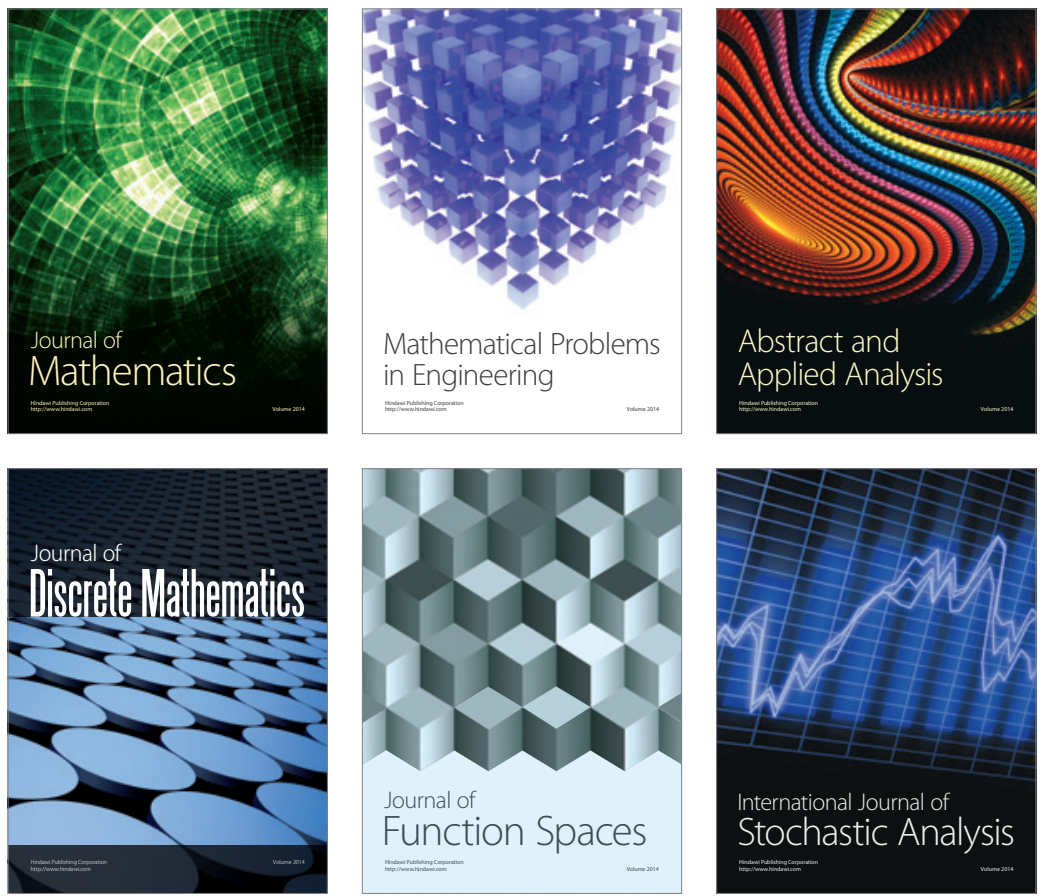

Journal of

Function Spaces

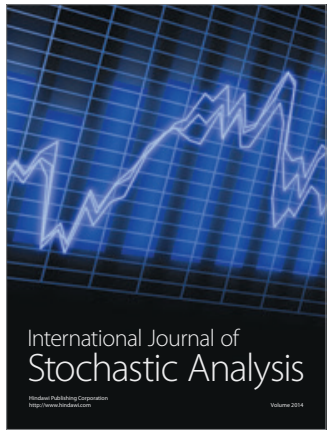

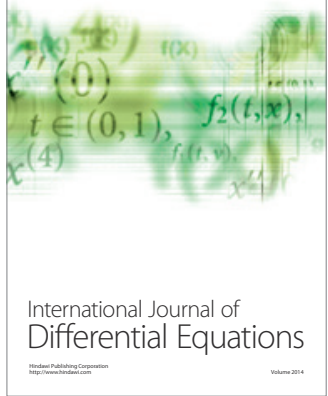
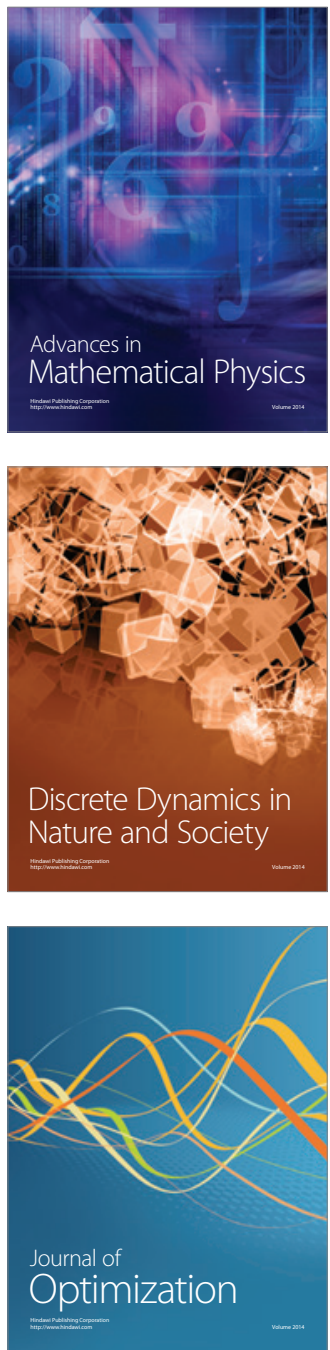